\title{
VOCES OCULTADAS, VOCES ESCUCHADAS: GÉNERO Y VIOLENCIA, UN BINOMIO A ESTUDIAR
}

\author{
GUILLERMINA DÍAZ PÉREZ \\ NATALIA IX-CHEL VÁZQUEZ GONZÁLEZ \\ Universidad Autónoma del Estado de México (México)
}

En el diálogo que mantenía Margarita Riviére con Salvador Giner, ella afirmaba que «los periódicos y las televisiones hablan de malos tratos y nos quedamos escandalizados: ¿es que no los había antes? Claro que los había, pero el hogar y sus mitos lo tapaban todo» ${ }^{1}$.

En México, la violencia intrafamiliar ha sido poco estudiada, por lo que hay una escasez de información acerca de las formas que asume en los distintos grupos sociales y las repercusiones de su práctica en otras esferas del comportamiento de los individuos ${ }^{2}$. Aún así, no cabe duda de que la violencia contra las mujeres en el ámbito doméstico tiene dimensiones sociales, ocupando un lugar importante en los índices de morbilidad y mortalidad femeninas. Ejemplo de ello es que, "en el caso concreto de las mujeres, la mayoría de las muertes por homicidio ocurren en el hogar y su pareja es la persona más frecuentemente involucrada en ese desenlace violento ${ }^{3}$. De ahí que, a medida que la violencia hacia las mujeres se haga visible, en ese mismo sentido, se apoyará la construcción de una cultura de paz.

Las presentes líneas tienen como finalidad presentar un ejemplo de la sensibilidad de algunas instituciones para hacer visible el problema de la violencia hacia las mujeres e incidir en las políticas sociales para mejorar la atención integral de las mujeres afectadas. La preocupación, por parte de funcionarias de la Legislatura Local del Estado de México, ante la existencia de violencia intrafamiliar ejercida sobre las mujeres que habitan la zona norte del municipio de

1. RVIIÉRE, Margarita y Giner, Salvador: Mujeres y Hombres. La impía relación, Madrid, Espasa, 1999, p. 19.

2. Arverra, Orlandina et. al.: "Familia y género en el análisis sociodemográfico», en Brígida García (coord.): Mujer, género y población en México, México, Colmex, 1999, p. 248.

3. Cómez-DANTES, Héctor et. al. : "La violencia en las mujeres usuarias de los servicios de salud en el IMSS y la SSA", Revista de Salud Pública en México, 48, suplemento 2 (2006), p. 280.

Feminismo/s, 9, junio 2007, pp. 139-151 
Toluca (zona que tiene un alto índice de ascendencia indígena) permitió evaluar la viabilidad para implantar un albergue orientado a su atención.

\section{LA VIOLENCIA}

Los supuestos bajo los cuales se plantea la relevancia de estudiar la violencia hacia las mujeres pueden encontrarse en lo estipulado en la IV Conferencia Mundial sobre las Mujeres ${ }^{4}$, donde se reconoce que las mujeres están sometidas, de manera creciente, a maltratos físicos, psicológicos y sexuales. Esta violencia se concreta de diversas formas: violaciones, abusos, acoso e intimidación sexual, tráfico de mujeres y prostitución forzada. Por lo general, todos estos actos contribuyen a mantener a las mujeres en una situación que les impide gozar de derechos y de libertades en pie de igualdad con los hombres.

Dos ámbitos donde se manifiestan de manera peculiar y patente las agresiones de los hombres contra las mujeres son el doméstico y el laboral. En el ámbito familiar las mujeres sufren condiciones de desigualdad, iniquidad, discriminación, malos tratos, exclusión social y desvalorización en su relación con los hombres. Situaciones que, en la mayoría de las ocasiones, quedan ocultas 5 .

La Encuesta Nacional de las Dinámicas de las Relaciones en los Hogares 2003, señala que, en México, el 46,6 \% de las mujeres mayores de 15 años que viven con sus parejas reportó haber sufrido alguna forma de violencia durante los doce meses previos al momento de las entrevistas ${ }^{6}$. De igual forma, en la Encuesta Nacional de Violencia contra las Mujeres, realizada por la Secretaria de Salud, el 25,8 \% de las mujeres encuestadas afirmaron haber experimentado en su vida una relación violenta ${ }^{7}$.

Según la Comisión Nacional de la Mujer de México ${ }^{8}$, las manifestaciones de la violencia intrafamiliar pueden presentarse de la siguiente manera:

- Violencia corporal, como, por ejemplo, bofetadas, patadas, empujones, amenazas, golpes con objetos, estirones, arañazos, intentos de estrangulamiento, arrojar sustancias, ensuciar el cuerpo de la otra persona, encerrarla, echarla de la casa o del automóvil, perseguirla dentro de la casa o fuera de ella, despertarla constantemente.

4. Para más información puede consultarse: www.un.org/esa/gopher-data/conf/fwcw/off/a--20.en

5. OIVEIRA, Orlandina: Op. cit., p. 250.

6. ENCUESTA NACIONALSOBRE LA DINÁMICA DE LAS RELACIONES EN LOS HOGARES 2003, México, INEGI, 2004 en http://cedoc.inmujeres.gob.mx/documentos_download/100758.pdf, p. 41.

7. INSTITUTO NACIONAL DE SALUD PÚBLICA: Violencia contra las mujeres. Un reto para la Salud Pública en México. Informe Ejecutivo de la Violencia contra las mujeres, México, INSP-SSA, 2004, p. 65.

8. COORDINACIÓN GENERALDE LA COMISIÓN NACIONAL DE LA MUJER: Informe final de avances en el cumplimiento del Programa Nacional contra la Violencia Intrafamiliar 1999-2000, México, INMUJER, 2000. 
- Violencia psicológica, en forma de insultos, descalificaciones, despojo de los hijos, silencio calculado, acusaciones de infidelidad, desprecios, abandono, chantaje o humillaciones en general.

- Violencia sexual: Exigencia de tener relaciones sexuales sin importar el deseo de la otra persona, uso de pornografía obligando a otra persona a copiar modelos, usar sexualmente a una persona como descarga hostil, violación dentro o fuera del matrimonio, acoso sexual, abuso sexual infantil, incesto, prostitución forzada, estupro.

- Abuso económico: No prever necesidades de la familia, gastar sólo en sí mismo, revisar a la otra persona su billetera, quitarle bienes materiales, no dar pensión alimenticia, declararse insolvente sin serlo.

- Violencia social: Impedir a la otra persona que le acompañe a actividades sociales, humillarle en público, impedirle trabajar, impedirle estudiar, prohibirle o condicionarle amistades, impedir o condicionar la visita a sus familiares, obligarle a consultar sus médicos o psiquiatras, internarle sin su consentimiento.

Al analizar dichas formas de violencia será necesario abordar el estudio del contexto familiar y tener en cuenta que, en muchas ocasiones, encontraremos todo un proceso de enmudecimiento por parte de las víctimas. La atención al entorno familiar permitiría conocer, en todo caso, si el dominio masculino se impone mediante la violencia física o psicológica, o es aceptado y asumido como legítimo por parte de los miembros de la familia, de tal suerte que ni siquiera se considere una manifestación de violencia. Así, por ejemplo, los abusos pueden afectar las creencias de las víctimas acerca de ellas mismas y de terceros, siendo difícil para ellas iniciar una conversación acerca de la violencia de género en sus vidas. La mujer que sufre violencia puede percibirse a sí misma como alguien que no tiene posibilidades de salir de la situación en la que vive e incapaz de valerse por sí misma ${ }^{10}$. En el siguiente cuadro, que presenta el Fondo de Naciones Unidas para la Población ${ }^{11}$, puede verse un ejemplo claro de ello:

9. CORSI, Jorge: Violencia Familiar. Una mirada interdisciplinaria sobre un grave problema social, Buenos Aires, Paidós, 1994, pp. 65-67.

10. Ibíd, p. 59.

11. FONDODE POBLACIÓN DE LAS NACIONES UNIDAS: Un enfoque práctico de la violencia de género. Guía programática para proveedores, Nueva York, FNUAP, 2001, p. 62. 


\begin{tabular}{|l|l|l|}
\hline $\begin{array}{c}\text { Qué comunica el atacante } \\
\text { a la sobreviviente }\end{array}$ & \multicolumn{1}{|c|}{$\begin{array}{c}\text { Qué interpreta la } \\
\text { sobreviviente }\end{array}$} & \multicolumn{1}{|c|}{$\begin{array}{c}\text { Cuáles son los efectos } \\
\text { sobre la sobreviviente }\end{array}$} \\
\hline Te pego porque te amo & El amor es así & $\begin{array}{l}\text { Confusión, reiteración de sus } \\
\text { experiencias como víctima }\end{array}$ \\
\hline $\begin{array}{l}\text { Tú tienes la culpa de que yo te } \\
\text { maltrate }\end{array}$ & Soy mala y tengo la culpa & $\begin{array}{l}\text { Autoculpa, confusión, } \\
\text { desamparo }\end{array}$ \\
\hline Nadie te amará como yo & $\begin{array}{l}\text { Sin él, estaré sola por el resto } \\
\text { de mi vida }\end{array}$ & $\begin{array}{l}\text { Dependencia, temor de } \\
\text { marcharse }\end{array}$ \\
\hline Lo hago por tu propio bien & $\begin{array}{l}\text { Otros saben qué es bueno } \\
\text { para mí }\end{array}$ & \begin{tabular}{l} 
Dudas acerca del propio juicio \\
\hline $\begin{array}{l}\text { Tú no eres dueña de tu } \\
\text { cuerpo, el dueño soy yo }\end{array}$
\end{tabular} No tengo control sobre lo que \\
otros me hacen & $\begin{array}{l}\text { Deficientes límites personales, } \\
\text { reiteración de sus experiencias } \\
\text { como víctima }\end{array}$ \\
\hline $\begin{array}{l}\text { Si hablas de esto, nadie te } \\
\text { creerá }\end{array}$ & Estoy sola y a nadie le importa & Silencio \\
\hline
\end{tabular}

De esta forma, la familia, como grupo base primario de los procesos de socialización, puede mostrar espacios de equidad y solidaridad, o, por el contrario, reproducir esquemas caracterizados por la desigualdad en las oportunidades y el desequilibrio en los accesos a los recursos. Asimismo la unidad familiar puede faltar a sus metas ideales: promover lazos de afecto y de unión social; facilitar el desarrollo de la identidad personal, familiar y social; promover la realización creativa de sus miembros y beneficiar su entrenamiento de participación social ${ }^{12}$.

La violencia en la familia no depende del nivel socioeconómico, ni de la edad, ni del grado de instrucción de las personas que la padecen. Dar preferencia a alguna de estas variables sería cegar la oportunidad de analizar y entender los mecanismos a partir de los cuales se están generando y reproduciendo esquemas de violencia sobre la mujer. Aunque no por ello se tenga que dejar de lado que existe una relación estrecha entre desigualdad social y violencia.

Establecer la relación entre violencia, familia y género, y estudiar la violencia desde el seno mismo de la familia permitiría, en todo caso, que las mujeres hablaran de cómo ellas viven la violencia, no sólo para que sepamos qué está pasando, y a quién le está pasando, sino más bien para saber cómo están pasando las cosas, y por qué están pasando ${ }^{13}$.

\section{EL ESCENARIO}

De acuerdo con los datos existentes, la prevalencia de la violencia de pareja es de $21,5 \%$ para el territorio nacional. Dependiendo del tipo de violencia, los

12. Vazouez, Natalia y Figueroa, José G.: «Pobreza y Género en el Contexto Mexicano», en José María Tortosa (dir.): Pobreza y perspectiva de género, Barcelona, Icaria, 2001, pp. 167-174.

13. INSTITUTO NACIONAL DE SALUD PÚBLICA: Violencia contra las mujeres. Un reto para la Salud Pública en México. Informe Ejecutivo de la Violencia contra las mujeres, México, INSP-SSA, 2004, p. 111. 
porcentajes se distribuyen de la siguiente manera: un $19,6 \%$ violencia psicológica, 9,8\% física, $7 \%$ sexual y $5,1 \%$ económica ${ }^{14}$.

Según la encuesta Nacional de Violencia Familiar ${ }^{15}$ que se levantó en la Zona Metropolitana de la Ciudad de México en 1999, en un 33 \% de los hogares existe algún tipo de violencia, maltrato emocional, intimidación, abuso físico o sexual. De acuerdo con los datos que proporciona dicha encuesta, de los 4,3 millones de hogares del área metropolitana de la Ciudad de México, uno de cada tres sufre algún tipo de violencia intrafamiliar. Según esta misma encuesta, los miembros de la familia más agresivos son el jefe de la familia $(49,5 \%)$ y la cónyuge $(44,1 \%)$, mientras que las víctimas más frecuentes en todos los tipos de maltrato fueron las hijas e hijos $(44,9 \%)$ y la cónyuge $(38,9 \%)^{16}$.

Los resultados de esta encuesta afirman que el maltrato emocional constituye la agresión más frecuente, en tanto que ocurre en un $98,4 \%$ de los casos, mientras que la intimidación se manifiesta en un $16 \%$, la violencia física en un $15,5 \%$ y el abuso sexual en un $14 \%{ }^{17}$.

Los resultados revelan que sólo solicitaron ayuda 14 de cada 100 hogares $(14,4 \%)$ en donde se registraron actos de violencia. Los tipos de apoyo más requeridos fueron el psicológico y el de la iglesia. En este sentido, la Encuesta Nacional de Violencia contra las Mujeres realizado por el Instituto de Salud Pública muestra que el intento de resolver el problema de la violencia conyugal puede comenzar después de años de maltrato y, cuando las mujeres lo deciden, se combinan dos tipos de búsqueda: los informales (personas de su entorno social inmediato, generalmente amigas), y los formales (diversas instancias de atención) $)^{18}$.

Por otra parte, de acuerdo con Guadalupe Cortés, un número importante de la población mexicana de 18 años y más años aceptan afirmaciones del tipo: "los gritos y los insultos son necesarios para resolver los problemas de la familia». Este dato sirve para mostrar que esta violencia no es asunto exclusivamente de corte biológico, sino que es una construcción social que se aprende, aprehende (interioriza y simboliza), y reproduce culturalmente, y que, en ocasiones, se asume como cuestión de "costumbre»"

En el caso concreto del presente trabajo, la investigación se realiza en la Zona norte de la ciudad de Toluca (Estado de México), la cual se compone de tres delegaciones: San Pablo Autopan, San Andrés Cuexcotitlan y San Cristóbal Huichochitlan. Se trata de una franja con un alto índice de marginación ${ }^{20}$ y su

14. Ibíd., p. 67.

15. INSTITUTO NACIONAL DE GEOGRAFÍA E INFORMÁTICA: La violencia Intrafamiliar. Encuesta 1999, México, INEGI, 2000.

16. Ibíd., pp. 50-53.

17. Ibíd., p. 15.

18. INSTITUTO NACIONAL DE SALUD PÚBLICA: Op. cit., pp. 30-31.

19. CORTÉS, Guadalupe: «Pareja y violencia», en Blanca Aguilar y Martha Tapia (coords.): La violencia nuestra de cada día, México, Plaza y Valdés, 2006, p. 129.

20. CONAPO: Índices de marginación 2000. Puede consultarse en la siguiente dirección web: www. conapo.gob.mx/publicación/indices/pdfs/003.pdf, p. 29. 
población la constituyen, principalmente, hombres y mujeres de ascendencia otomí ${ }^{11}$. De acuerdo con datos proporcionados por la Procuraduría General de Justicia del Estado de México, las denuncias recibidas en estas localidades dejaban sospechar la existencia de altos niveles de violencia intrafamiliar, ya que durante 2003 las denuncias sobre lesiones y denuncia de hechos alcanzaron los más altos exponentes. Sin embargo, en el momento de rastrear datos más precisos la tarea no rindió los frutos esperados, ya que no se ha aplicado un sistema adecuado que pueda dar cuenta de ello, sobretodo en el ámbito municipal y delegacional.

Partiendo de esta realidad, en un primer momento se aplicó un cuestionario donde las preguntas giraban en torno a cinco tipos de violencia (social, física, psicológica, económica y sexual). La finalidad de dicha aplicación era analizar la viabilidad de la técnica e instrumento, ya que desde un principio se sospechaba de las dificultades que su uso implicaba.

La población muestral la componían mujeres que vivieran en pareja y que fueran mayores de 18 años. La aplicación se realizó durante dos fines de semana, en un horario de 11:00 a 14:00, en las plazas delegacionales de cada una de las comunidades. Previamente se capacitó para su aplicación a seis estudiantes mujeres de la Licenciatura en Comunicación. Se aplicaron un total de 28 cuestionarios. La aplicación dio como resultado lo que se sospechaba en un principio: las mujeres, transeúntes de la plaza, regularmente se hacían acompañar de su pareja y era imposible aplicarles el instrumento. Las pocas mujeres que transitaban por las plazas y podían dar respuesta, afirmaban tener un marido o pareja tranquila y nada violento. Sin embargo, al considerar otras preguntas como: ¿Su esposo la vigila?, ¿Su esposo se enoja muy fácilmente en la calle?, ¿Quién es el que aporta el dinero a su casa?, ¿Su marido le da (lo de) su gasto?, las respuestas remitían a situaciones vivenciales de violencia.

De ahí que se pensara en la necesidad de una técnica e instrumento a partir de los cuales se pudiera comprender la violencia desde su significación, ya que las respuestas en los pocos cuestionarios aplicados dejaban ver a todas luces que la violencia, más que un hecho, implicaba una interpretación, y, por tanto, para su entendimiento era necesario distinguir las dinámicas de su desarrollo y sus consecuencias. Es así que, en un segundo momento, se optó por el trabajo etnográfico como proceso de descripción. Esta opción metodológica permite, por una parte, ver cuáles son las interpretaciones que los actores hacen de su propia acción (en este caso las mujeres); y, por otra, hacer una reinterpretación de la interpretación que hacen los actores, a la luz de los supuestos conceptuales que ordenan la investigación.

Para la realización de este segundo momento -el diseño e instrumentación del trabajo etnográfico-, se solicitó el apoyo de la Facultad de Antropología

21. Ver Fernández, Patricia et.al.: "Estimaciones de la Población Indígena en México», en CONAPO: Situación Demográfica 2002, consultada en http://www.conapo.gob.mx/publicaciones/2002/13.pdf., pp. 175-181. 
de la UAEM bajo la colaboración de dos profesoras y la participación de 21 estudiantes de la Licenciatura en Antropología. Para la realización del diagnóstico era importante el trabajo de campo con estancia en la comunidad, por ello se optó por la observación participante y directa en las tres comunidades, junto con la realización de entrevistas a mujeres. La selección de entrevistadas respondía a los siguientes criterios: mujeres que tuvieran relación de pareja, mayores de 18 años y que accedieran a participar.

En primer lugar, se seleccionaron los barrios / colonias o sectores de las comunidades para abarcar la zona objeto de estudio. Una vez hecho, a partir de una primera observación, se seleccionaron los hogares e informantes para la aplicación de las entrevistas, dos por cada barrio.

Vale la pena aclarar que, en las tres comunidades estudiadas, es muy frecuente la endogamia al igual que la residencia trigeneracional. En este caso, se trata de familias extensas que no rebasan los ocho miembros por vivienda. En tales núcleos familiares, cuando la madre sale a trabajar, la abuela suele quedarse al cuidado de los nietos, por ello, en repetidas ocasiones, se escucha que los niños llaman «mamá» a quien en realidad es su abuela. Igualmente, hay mujeres que desde los 35 años son abuelas, es decir, en tres décadas ya existen tres generaciones; si bien en la localidad también hay mujeres que tienen más de 75 años, lo que representa un índice de longevidad importante. La residencia es patrilocal.

El rezago educativo es mucho mayor en las mujeres que en los hombres y la jefatura de los hogares tiende a ser masculina. En la comunidad se acostumbra el robo de la novia, el cual va acompañado "del perdón», un pago que se hace a los padres de la novia por medio de canastos de comida llamado «chiquigüite».

Las mujeres, después de haber terminado el trabajo diario, se dedican a realizar costuras para su hogar, o al tejido de algunas partes de los sombreros, o preparan nizcomel (cociendo el maíz para hacerlo masa) para elaborar tortillas el día siguiente. De esta forma, cada mujer está en su hogar, dificultando que puedan reunirse, aunque sólo fuera para hablar. Únicamente cruzan algunos comentarios en las mañanas, cuando llevan a sus hijos a las escuelas, o cuando van a comprar a las tiendas o a las verdulerías. En estas ocasiones, algunas se quedan un tiempo hablando, otras únicamente se saludan y regresan a continuar con los quehaceres de su casa y tener lista la comida para cuando lleguen el marido y los hijos.

La bicicleta es un medio de transporte bastante difundido que utilizan tanto hombres como mujeres de todas las edades, sin embargo podemos observar una serie de diferencias en su utilización. Las jóvenes que son solteras y no tienen novio conducen su propia bicicleta cuando van por las tortillas o a la recaudería. En cambio, en el caso de aquellas que tienen novio, debe ser él quien conduzca y acondicione la bicicleta para que su novia pueda viajar en la parte de atrás o en el cuadro de la misma. Finalmente, las mujeres que están casadas no suelen montar en bicicleta, pero, en caso de que sea así, su esposo debe ser quien maneje. 


\section{LOS RESULTADOS}

\subsection{Violencia social}

Las mujeres de la comunidad, en términos generales, no cuentan con niveles de estudios superiores al básico o secundario. Suelen dedicarse al trabajo doméstico y no trabajan fuera de este ámbito.

"Las labores del hogar son realizadas solamente por las mujeres; las hijas apoyan a sus madres y les ayudan con algunos quehaceres como barrer, trapear, y lavar. Todas las decisiones concernientes al ámbito domestico como la comida, despensa, jabón, etc., son tomadas por el sexo femenino. Los hijos varones por lo regular trabajan ayudando al padre o en actividades concernientes al campo ${ }^{22}$.

Si bien en las respuestas las entrevistadas dicen contar con un «marido bueno» o un "buen marido", el discurso señala que existen limitaciones a la hora de visitar a la familia o amigos; incluso la realización de compras suele realizarse en compañía de los esposos. Esto último es considerado por las mujeres como un síntoma de galantería, cuando también puede interpretarse como un control del gasto y de la vida cotidiana por parte de los hombres; $y$, simbólicamente, ejemplificaría la aparente imposibilidad, por parte de la mujer, de tomar decisiones correctas.

«El tiempo de esparcimiento se presenta cuando el marido tiene el día libre, y la invita junto con su familia a visitar lugares diferentes, como es el caso de la señora Gloria que vende tortillas y tiene dos hijos, quien comenta que los sábados y domingos o cuando su esposo tenía tiempo, salía la familia a lugares como Tenancingo, Ixtapan de la Sal o Tonatico, y ahora ya no porque uno de sus hijos estudia, y su hija trabaja en la fábrica» ${ }^{23}$.

El espacio de la casa en el que la mujer puede ejercer cierto control es la cocina, que es, relativamente, un espacio privado y de confinamiento. La mujer asume que "lo lógico", "natural», es que ella sea la responsable y la única que debe estar ahí, al grado de legitimar que el esposo no tenga ingerencia en las actividades culinarias.

La violencia se naturaliza hasta el punto de opacar la existencia de otros espacios, desde donde la mujer pueda ejercer sus potencialidades. Así, por ejemplo:

«La Señora Enedelia, quien en 30 años de matrimonio no ha obtenido ningún tipo de apoyo económico por parte de su esposo, para mantener a sus hijos ha tenido que emplearse, en un primer momento, lavando ropa ajena, después, a través de la cría y venta de guajolotes, pollos, patos, puercos; actividades mismas que le han permitido sostener los gastos de sus 5 hijos, quienes sólo asistieron a primaria o secundaria, según los recursos que tuviera... No ha dejado a su esposo porque considera que iba a dejar a sus hijos desprotegidos. El último hijo la ha retenido al lado de su esposo; fue

22. UAEMex: Diagnóstico para Albergue de mujeres violentadas, Trabajo de investigación sin publicar, Toluca, 2005, p. 101.

23. Ibíd., p. 156. 
un embarazo no previsto en el cual el anticonceptivo que usaba no funcionó. Sólo espera que tenga la edad suficiente para que pueda entender la situación y poderse marchar a una casa que construyó por medio de los ahorros del dinero que sus hijos al trabajar le dan y de un terreno que fue la herencia de su padre al morir» ${ }^{24}$.

\subsection{Violencia de abuso económico}

La jefatura de hogar se suele otorgar de acuerdo a la persona que provee los recursos financieros para el sustento del hogar; para el caso específico de esta zona, la jefatura de hogar reside en el hombre, quien es el que sale a trabajar mientras las mujeres se quedan al cuidado del hogar.

En este sentido, el hombre determina el presupuesto familiar, desde el momento en que él decide acompañar a la esposa a realizar la compra de la despensa y no perder el control sobre tal presupuesto. Por supuesto, en la mayoría de las entrevistas salió a colación que el hombre es comprensivo, porque paga, no sólo la comida, sino los gastos generales que implican mantener una casa. Sin embargo, el hecho de que el hombre sea la fuente de ingresos del hogar, le "da el derecho» a determinar cómo se distribuye el gasto.

La observación, de igual manera, muestra que el hecho de que sea el hombre el que determine la manera de distribuir el presupuesto familiar orienta la toma de decisiones de manera menos justa e igualitaria. Como ejemplo de ello, a medida que se avanza en el sistema educativo, las niñas tienen mayor índice de deserción que los hombres, y ya en la secundaria o en el sistema de bachilleres hay más población de varones que de mujeres. La respuesta de las mujeres es que los espacios privilegiados para ir a la escuela los tiene el hombre, y quien determina las mejores decisiones para la familia es el jefe de familia, sobretodo cuando los recursos económicos son escasos.

A pesar de ello, se observó y se puso de manifiesto, a través de las entrevistas, que algunas mujeres quisieran para sus hijas mejores espacios de crecimiento y desarrollo, así como que tuvieran mayor acceso a una educación formal o que las hijas tuvieran el "privilegio» de ir a la escuela sin necesidad de llevar una doble jornada (escuela-labores del hogar).

En el caso en el que las mujeres tienen que mantener a la familia debido a que el hombre no les da dinero para el sustento, buscan alternativas, ya sea lavando o realizando trabajo doméstico en casas ajenas a la suya. La distribución del poco dinero que llevan a casa suele realizarse de manera solidaria y equitativa, procurando cierta igualdad en la distribución de recursos y oportunidades: "yo no voy a darte educación a ti y a matar de hambre a tus hermanos» ${ }^{25}$, expresaron algunas de las entrevistadas. 


\subsection{Violencia sexual}

Gran parte de las mujeres que participaron en el estudio manifestaron la existencia de violencia sexual, en algunos de los casos asociada a problemas de alcoholismo por parte del marido. Las mujeres usan expresiones como: «abusó de mí", "los hijos llegaron cuando yo no lo quería» ${ }^{26}$, lo que puede suponer que no importó el deseo de la mujer a la hora de mantener relaciones sexuales y se vio limitada la posibilidad de controlar su capacidad reproductiva.

Independientemente de la relativa asunción de la violencia como algo natural, es importante resaltar que las mujeres que tienen que mantener a su familia plantearon, a lo largo del trabajo etnográfico, la posibilidad de romper el ciclo que les provoca dolor, angustia y miedo, a través de dejar al marido y llevarse a los hijos o de romper toda relación con la pareja y la familia de ésta:

"Una vez discutimos. Lo saqué de la tienda para que no se emborrachara y me pegó, yo estaba embarazada y me dio una patada e iba a abortar. Mi suegra me llevó al doctor, me pusieron unas ampolletas grandísimas para que lo detuviera y no lo aborté, pero antes ya había tenido 2 abortos, que fueron porque me pegaba. Sólo me pegaba cuando estaba borracho, en juicio era muy amable, cuando estaba borracho era bien grosero y bien malo, yo sufrí bastante con él, pero Diosito se lo llevó y yo estoy aquí. Se emborrachaba y jamás se calmaba, así empezó desde que empecé a estar con él, desde que era chavo sólo tomaba alcohol todo el tiempo, yo no me di cuenta ... yo ya me quería ir pero ¿mis hijos con quién se iban a quedar?, le dije a mi suegra yo ya me voy ¿como voy aguantar los trancazos...? yo todo hacía, todo aguantaba, él me decía 'si quieres vete', él tenía a otra mujer...»²7.

\subsection{Violencia psicológica}

Lo más sobresaliente de este apartado reside no sólo en el hecho de haber encontrado una abundante presencia de hechos tales como insultos, descalificaciones, la atadura al marido sólo por los hijos, desprecios, abandonos, chantajes y humillaciones, sino acaso más por el hecho de saber que las diferentes manifestaciones de la violencia han provocado, en muchas de estas mujeres, un miedo hacia la figura del marido, al grado de estar confundidas al momento de direccionar sus acciones. Aún así, la ambigüedad y el hecho de que se tenga la posibilidad de tener un trabajo fuera de la casa también ha permitido que dichas mujeres piensen en la posibilidad de romper este círculo.

Si bien se observa un elevado desgaste emocional en las mujeres al vivir la contradicción que marcan, por una parte, las pautas familiares, culturales y sociales, y por otra, la toma de decisiones personales de no permitir y legitimar un espectro mayor de violencia, también se observa un contexto muy propicio para poder instalar e instrumentar un albergue temporal para mujeres violentadas, en tanto que los discursos que se dejan entrever señalan espacios para poder 
ejercer el empoderamiento, y si bien no signifique romper con la violencia, por lo menos sí con los procesos de feminización de la pobreza.

No es de extrañar, en este sentido, que la posibilidad de trabajar fuera del hogar sea una fuente importante de empoderamiento para las mujeres, en tanto descubren su capacidad de trabajar y ganar dinero. Igualmente facilita el hecho de contar con mayores redes sociales y oportunidades de capacitación. En los distintos grupos de edad, el empoderamiento se hace manifiesto a través de frases como "abrir los ojos»" ${ }^{28}$, alusión que funciona como sistema de interpretación cuando se revela un cambio de actitud ante la violencia.

\subsection{Violencia física}

Este tipo de violencia parece ser bastante frecuente en las mujeres entrevistadas, si bien, puede significarse de diversas maneras, al grado de llegar a legitimar, en determinadas ocasiones, su uso como necesario.

De acuerdo con los resultados encontrados, a medida que las mujeres pueden hablar de los vaivenes del mercado laboral, y no sólo de los espacios privados en los que se encuentran confinadas, parecen tener una visión más crítica en cuanto a la significación que se le puede otorgar a los golpes.

En este sentido, a lo largo del estudio se encontraron casos graves de violencia física, en muchas ocasiones cuestionados por las mujeres:

"Me pegaba en mi cabeza, mi cintura, era un hombre que no tenía nada de compasión, yo no me defendía ¿cómo me iba a poner [enfrentar] con un hombre bien borracho?, yo creo que me mataba, hasta me daba miedo. Era una tonta, se dejaba uno... a mis hijos sí les pegaba cuando venía borracho; llegaba, gritaba, maldecía, hasta harto miedo; bien borracho, llegaba y al otro día se iba a emborrachar...Era una vida de perro porque no entendía, nunca me daba para el gasto...Él abusaba de mí, y él se enojaba, yo ya no quería; me pegaba nada más venía a lo que quería, no ya no, yo ya no me quedaba con él, me quedaba con mis hijas; el coraje que tenía, que tal si en eso me ahorcaba, entonces ya mejor me dejaba, todos (mis hijos) nacieron así de abuso, digiera usted un cariño, que me acariciara o así, no nada, nada más así..... ${ }^{29}$.

De esta forma, aquellos elementos de la violencia que han sido solidificados y cimentados por las estructuras sociales y culturales pueden empezar a desmontarse. Claro está que las víctimas de la violencia pueden llegar a reproducir la violencia en sus espacios relacionales y por tanto se hace necesario continuar trabajando para romper dichos ciclos de la violencia.

\subsection{El Albergue}

Los resultados y referencias sobre la violencia que se ejerce contra las mujeres desde el ámbito doméstico en la zona norte de la Ciudad de Toluca evidencian la necesidad de potenciar y fortalecer la posición social, económica y

28. Ibíd., p. 139.

29. Ibíd., p. 133. 
política de las mujeres, a través del reconocimiento de las propias capacidades y habilidades para ejercer influencia, poder y liderazgo en las relaciones sociales y actuar en función de ese reconocimiento. Por ello mismo, se asumió como conclusión general la viabilidad de la construcción de un Albergue para mujeres violentadas, que no sólo diera respuesta a las necesidades inmediatas, sino que fundamentalmente se asumiera como un compromiso institucional para modificar la condición y posición de las mujeres y lograr así un sistema sexo-género más equitativo, justo y solidario.

En su momento, la construcción del Albergue para mujeres violentadas daría respuesta a la necesidad de atención que requiere un problema de este tipo, que lejos de limitarse al ámbito doméstico, permea las condiciones estructurales, sociales e institucionales que se constituyen en caldo de cultivo. En este sentido, el Albergue se presenta como una oportunidad para comprender y actuar sobre la constitución de una serie de valores y símbolos fuertemente arraigados a los fenómenos de la violencia de la que son objeto las mujeres.

En tanto que se presenta como un espacio a partir del cual poder atender con criterios de justicia y equidad social el problema de la violencia, también puede ayudar a desarrollar investigaciones que analicen cómo determinados grupos sociales leen, significan y utilizan la violencia, en aras de poder actuar sobre la misma.

$\mathrm{Si}$ bien es cierto que a lo largo del presente escrito quedan esbozadas las maneras en las que las mujeres significan y viven la violencia, también es cierto que se vislumbran espacios a partir de los cuales el empoderamiento se hace patente. El Albergue, desde esta visión, deberá contar con un servicio integral que abarque desde lo médico hasta lo legal, pasando por aquellos servicios que ayuden a la mujer a alcanzar la autoestima personal y la recuperación de la dignidad como persona, de forma que pueda tomar conciencia del poder que tanto individual como colectivamente tiene; asumiéndose como agentes activos de su propio desarrollo.

El Albergue, en este entendido, no sólo debe dar respuesta a las necesidades inmediatas de una búsqueda de refugio ante situaciones de violencia, sino fundamentalmente ofrecer alternativas para detonar los procesos de empoderamiento y de cambio en las condiciones de su vida cotidiana. Es decir, debe plantearse desde una mirada inter, multi y transdisciplinar, para que en su instrumentación cuente con los servicios profesionales necesarios que lo constituyan como un servicio integral.

Es preciso que el Albergue se disponga como un recinto privado, a puertas cerradas, donde sea posible que la mujer se sienta segura, y que lejos de colocarla en situación de peligro sea un espacio donde aprenda que su persona tiene dignidad. Por ello mismo, deberá ser un espacio donde se sea testigo, se escuche y valide, se apoye, se actúe, se documente y sobretodo se dignifique a la mujer. 


\section{REFLEXIÓN FINAL}

Las líneas aquí vertidas dejan dos reflexiones generales. La primera de ellas tiene que ver con la manera en cómo se significa la violencia contra las mujeres. No hay que olvidar que la violencia se ha anclado en los procesos sociales, pero que, al mismo tiempo, convive, contradictoriamente, con un sentimiento opuesto y una necesidad de condenarlo.

Conviene entonces preguntarse cuáles son aquellos procesos de significación que permiten que la violencia hacia la mujer se legitime en las prácticas cotidianas como algo lógico y normal; cuáles y cómo se dan los procesos de victimización; cuáles y cómo se plantean las consecuencias psicológicas en las víctimas; cuáles y cómo pueden aprovecharse los sentimientos encontrados para que se fortalezcan en procesos de empoderamiento ${ }^{30}$; bajo el entendido de que el empoderamiento ${ }^{31}$ trabaja sobre tres niveles: el social, el político y el psicológico; y como tal, no es sólo un proceso personal y colectivo, sino que es el resultado mismo de dicho proceso.

La segunda reflexión tiene que ver con los espacios de visibilidad de la violencia y su consideración para las políticas públicas en aras de una sociedad más justa y equitativa. Aquí vale la pena argumentar que para hacer visible la violencia no basta sólo con indicadores a partir de los cuales se expresa, sino que se hace necesario presentar las manifestaciones a partir de las cuales se significa y simboliza la vida, los sentidos a partir de los cuales se buscan nuevos giros que conviertan las acciones silenciadas en acciones sostenedoras de las propuestas que hacen evidente la trama política, económica y social. La sensibilidad de las autoridades es importante ${ }^{32}$, pero también un factor valioso ha sido la presencia de las mujeres en el ejercicio profesional del periodismo escrito que han motivado el diálogo permanente y han incluido en la agenda mediática el tema de la violencia familiar y la violencia de género y que lejos de presentarse como mero espectáculo, forman parte de las políticas sociales. De igual forma, algunos grupos de mujeres indígenas han sido detonantes de movimientos sociales pacíficos que han imbricado en el sistema de relaciones simbólicas y han permeado el cambio cultural, motivando, entre otras cosas, el empoderamiento en otras mujeres que viven una condición violenta.

30. EnRíauez, Rocío:«Pobreza y hogares de jefatura femenina en México», en Gallardo (coord.): Los Rostros de la pobreza. El Debate, Tomo II, México, UIA-ITESO, 1998, p. 278.

31. Tortosa, José María (ed.): Pobreza y perspectiva de género, Barcelona, Icaria, 2001, p. 10.

32. Gutiérrez, Ma Trinidad et. al. (comps.): Género en el Desarrollo, México, Cidhal, 2006, p. 62. 